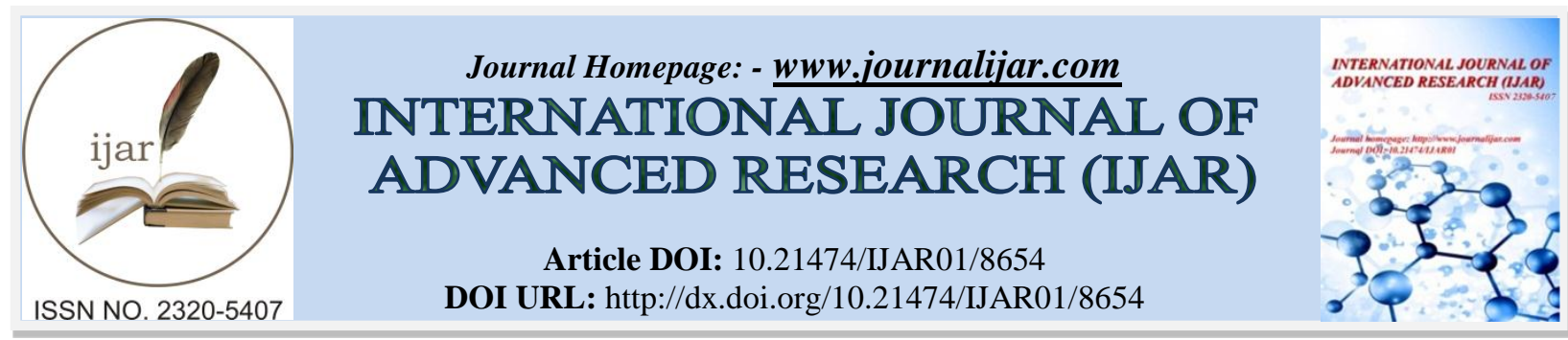

RESEARCH ARTICLE

\title{
EFFECT OF KINESIO TAPE ON ANKLE JOINT POSITION SENSE AND EVERTORS PEAK TORQUE IN FOOTBALL PLAYERS FOLLOWING EXERCISE INDUCED FATIGUE.
}

Moayad Dhahi, $\mathrm{MSc}^{1}$ and Mohammed S. Abdelsalam $\mathrm{PhD}^{1,2}$.

1. Department of Physical Therapy, College of Applied Medical Sciences, Imam Abdulrahman Bin Faisal University, Dammam, KSA.

2. Department of Physical Therapy for Musculoskeletal disorders and its Surgery, Faculty of Physical Therapy, Cairo University.

\section{Manuscript Info}

…......................

\section{Manuscript History}

Received: 09 January 2019

Final Accepted: 11 February 2019

Published: March 2019

Key words:-

Kinesio taping, Ankle Sprain, Joint position sense.
Abstract

Aim: This study aimed to assess whether the application of Kinesio taping (KT) of peroneus longus muscle would maintain ankle joint position sense (JPS), and evertors peak torque (EPT) in football players following exercise-induced fatigue. Methods: Forty football players from $1^{\text {st }}$ and $2^{\text {nd }}$ divisions of professional Saudi football leagues volunteered in this study. Volunteers were randomly assigned to Kinesio tape group (KTG) or control group (CG). Volunteers' ankle JPS, at $10^{\circ}$ inversion and full range of motion of inversion $-5^{\circ}$ (FROM $5^{0}$ ), and EPT were evaluated at baseline and following fatigue protocol. Volunteers in KTG were taped after baseline evaluation. Results: this study showed that volunteers in KTG recorded a non-significant difference of absolute angular error (AAE) post fatigue compared to baseline for both target angles. While volunteers in $\mathrm{CG}$ showed significantly higher AAE post-fatigue compared to baseline for both target angles. Further, KTG showed a significantly lower AAE post fatigue at target angle $10^{\circ}$ inversion compared to CG. EPT was significantly lower post fatigue compared to baseline in KTG and CG. Further, post fatigue EPT was non- significantly different between KTG and CG. Conclusion: KT application might help in maintaining JPS, but not EPT following exercise-induced fatigue in football players.

Copy Right, IJAR, 2019,. All rights reserved.

\section{Introduction:-}

The ankle joint is one of the most commonly injured parts of the body for individuals who participate in competitive activities, accounting for approximately 30\% of all sports related injuries (Hootman et al., 2007; Martin et al., 2013; Waterman et al., 2010). Ankle injury information pointed out that ankle sprains were the most common ankle injury in 33 different sports (Fong et al., 2007). Further, lateral collateral ankle sprains were reported in $77 \%$ of ankle ligaments injuries (Fong et al., 2009). The high incidence of ankle sprains in sports called for prophylactic measures where several interventions had been recommended (Porter and Rushton, 2015).

Kinesio tape (KT) is an adhesive tape frequently used in sports to serve different purposes including pain reduction and restoring range of motion (ROM) (Gonzalez-Iglesias et al., 2009), muscle facilitation and inhibition (Fayson et 
al., 2015; Subasi et al., 2016), and improving of joint function, and stability (Chang et al., 2010; Hsu et al., 2009). Further, proprioception is suggested to be enhanced due to stimulation of cutaneous mechanoreceptors during the KT application to the skin (Chang et al., 2010). Fatigue of supporting muscles is one of the reported factors that increases the risk of inversion ankle sprains (Gabbett, 2000). Some level of fatigue will be expected and achieved during athletic practice or competition (Ament and Verkerke, 2009). It is reported that neuromuscular control of the ankle is affected during fatigue period influencing ankle dynamic stability (Woods et al., 2003). Likely, the contribution of fatigue in risk of inversion ankle sprains result from altering ankle joint position sense (JPS) (Mohammadi and Roozdar, 2010). Evertor muscles are of interest since ankle evertors help in preventing inversion injuries which is the most common mechanism of lateral ankle ligaments injuries (Willems et al., 2002; Wilson and Madigan, 2007)

Whether the suggested effects of KT on proprioception and muscle activity could counteract the effect of fatigue of ankle evertors is not clearly investigated. Accordingly, this study aimed to check the effect of application of KT to peroneus longus muscle on ankle JPS and EPT following exercise-induced evertor muscles fatigue.

\section{Methods:-}

This was a pre-post control group study. Forty uninjured football players from first and second divisions of Saudi professional football leagues volunteered to participate in the study. G*power software was used to calculate sample size at power of 0.8. Players were excluded from the study if they previously sustained skeletal injuries or had surgeries of the ankle and foot. In addition, if they had history of first time ankle sprain within the last 3 months, or complain of chronic ankle instability. Further, if they had any open wounds of the leg, ankle and foot, skin diseases, or known hypersensitivity to tape materials. Players who volunteered and were illegible to participate were allocated randomly to one of the two equal study groups; Kinesio tape group (KTG) or control group (CG). Random number generator (http://www.randomizer.org) was used to generate two unsorted independent sets of numbers between 1-40 without repetition. Every enrolled player was allocated to the set in which his order of enrollment appeared. Volunteers had explanation of the aim and procedures of the study. Volunteers signed an informed consent form to confirm their participation prior to data collection. The institutional review board of Imam Abdulrahman Bin Faisal University provided ethical approval for this study.

\section{Procedures:-}

Volunteers had one visit to the laboratories of department of physical therapy, college of applied medical sciences, Imam Abdulrahman Bin Faisal University, where the study was conducted. Each volunteer was introduced to the laboratory and had a brief explanation of the isokinetic dynamometer used in the study. Then, demographic data (age, weight, and height) were recorded. Afterwards, the volunteer started warm up for 10 minutes on treadmill and 5 minutes on bicycle ergometer in preparation to evaluation and fatigue protocol. Evaluations of ankle JPS and EPT at baseline and post fatigue as well as the fatigue protocol were carried out on Biodex multi-joint system 4 (Biodex Medical Systems, Shirley, New York, USA), and were the same for volunteers in both KTG and CG. Previous literature reported non significantly different JPS comparing dominant and non-dominant legs (Lephart et al., 1998; McLoda et al., 2009). Likewise, Dabadghav (2016) demonstrated non - significantly different isokinetic invertor/evertor strength between dominant and non- dominant legs. Accordingly, the right leg was selected for test in this study for all volunteers.

\section{Evaluation:}

\section{Evaluation of ankle joint position sense (JPS)}

Ankle JPS was evaluated using Biodex multi-joint system 4. Active angle- reproduction test, which is a valid and reliable test to measure the ankle joint proprioception (Drouin et al., 2004), was used in evaluation of JPS. Volunteers were positioned based on the manufacturer's guidelines for assessing JPS for ankle joint. Volunteer under evaluation sat on Biodex chair and examiner stabilized him by chest and waist belts. The right knee was positioned and fixed with a dynamometer attachment at $45^{\circ}$ knee flexion while his right thigh was supported and straps were used to stabilize of the leg. The tested foot was fitted with special foam shoe, placed on the footplate, and stabilized by two velcro straps that secured the foot to the ankle attachment (Fig 1).

First, the examiner explained test procedures to the volunteer. Two target positions were tested, 1) Active inversion to $\left.10^{\circ}, 2\right)$ active inversion full range of motion minus $5^{\circ}\left(\right.$ FROM $\left.-5^{\circ}\right)$, starting from neutral ankle position. A blind draw was used to randomize the order of testing for each volunteer. During the assessment, the volunteer was blindfolded to prevent any visual information from distorting the test. The volunteer performed a familiarization trial 
to adapt to the test setup before beginning the data collection. Then, measurements were conducted where the volunteer carried out a set of 3 repetitions for each target angle. Each repetition consisted of two consecutive steps; first, was a training repetition where the volunteer actively moved to the target angle where the dynamometer held the position for 10 seconds, and volunteer was asked to concentrate on this angle to reproduce it during the next step. The second step was the recording trial, where volunteer moved his foot to the position he perceived to be the target angle then he stabilized the platform. Volunteers were allowed 20 seconds rest between trials of the same target angle, and one minute rest between the two target angles. Absolute angular error (AAE) was used as outcome parameter. Average of AAE of the 3 recording trials of each target angle was used for analysis.

\section{Evertors peak torque (EPT)}

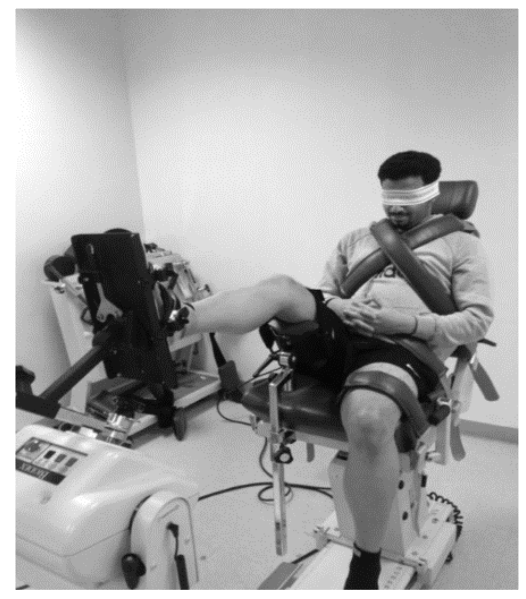

Fig 1: Testing ankle JPS

Biodex multi-joint system 4 was utilized to record peak torque of concentric ankle eversion for volunteers in both groups, before and after the fatigue protocol. The Biodex multi-joint system 4 is valid and reliable to measure the EPT (Tankevicius et al., 2013) The volunteer maintained the same position used for evaluation JPS on the Biodex multi-joint system 4. For familiarization with the isokinetic measure, each volunteer performed 3 consecutive submaximal inversion/eversion contractions with an angular velocity of $120 \% \mathrm{sec}$. Afterwards, during the isokinetic testing, volunteer's EPT was recorded from 3 consecutive maximal force at $60 \%$ sec. Examiner asked the volunteer to use safety button that allowed him to terminate the test at any time if he felt that it was too hard (Fu et al., 2008).

\section{Kinesio taping (KT) application}

The KT (Kinesio ${ }^{\circledR}$ Tex Gold ${ }^{\mathrm{TM}}$ FP) was used in this study. The KT was applied by the same therapist who well trained in applying KT. Tape was applied from insertion to origin, which was used to inhibit the muscles activity (Kase et al., 2010). During application, the volunteer rested in supine lying, and the length of the I-strip (2-inch in width) used in taping was taken as $2 / 3$ of the volunteer's leg length. The anchor of the tape started without tension from the lateral side of the base of $1^{\text {st }}$ metatarsal bone and lateral side of cuneiform bone, then over lateral border of the foot crossing the lateral side of cuboid (Gray, 2000). Then the ankle was plantar flexed and inverted to increase tissue tension on the lateral aspect of the ankle. The tape was stretched about $25 \%$ of its original length as it passed behind the lateral malleolus, all through the path of peroneus longus up to the head of fibula. (Kase, 2005) (Fig.2).

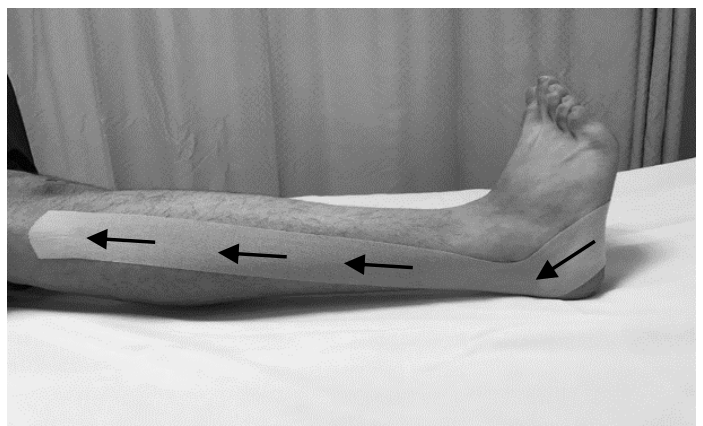

Fig 2: KT application Fatigue inducing exercises protocol. 
Volunteer performed the exercise protocol that aimed to induce fatigue on Biodex multi- joint system 4 using isokinetic mode. Preset angular velocity in direction of eversion was set to $60 \%$, while in inversion it was set to $400 \%$ s. This very high preset angular velocity in inversion direction was intended to provide very light resistance to invertor muscles. Thus, protocol targeted mainly evertor muscles fatigue. The repetitions of fatigue protocol were 50 cycles of eversion/inversion. Repetitions were set according to a previous study of ankle plantar flexion fatigue protocol (Larsson et al., 2006). Thus, it was considered that 50 repetitions were sufficient for the less bulky evertors. Volunteer's position was similar to that used in evaluation of isokinetic muscle strength. During the fatigue protocol examiner provided verbal encouragement to the players were encouraged to exert as hard pushing as possible and to reach the full range of motion during each repetition (McNair et al., 1996).

\section{Statistical Analysis}

Statistical package for the social sciences (SPSS) for windows 17.0 was used to do all the statistical analysis. Mean and standard deviations was given as descriptive statistics. For each dependent variable a mixed model ANOVA used for each dependent variable. The between factor variable was groups (KTG, CG). Within subjects factor was evaluation times (Baseline, post fatigue). $\mathrm{P}$ value was $<0.05$.

\section{Results:-}

Table (1) shows demographic characteristics for volunteers in the KTG and CG. The results show no statistical differences between volunteers in both groups concerning all recorded data at $(\mathrm{P}<0.05)$.

Table 1:- Volunteers' demographic characteristics

\begin{tabular}{|l|l|l|l|}
\hline \multicolumn{2}{|l|}{ Variables } & Groups & \multirow{2}{*}{ Independent t-test } \\
\cline { 2 - 3 } & KTG $(\mathrm{n}=20)$ & CG $(\mathrm{n}=20)^{\mathrm{a}}$ & \\
\cline { 2 - 3 } & Mean $( \pm \mathrm{SD})$ & Mean $( \pm \mathrm{SD})$ & P value \\
\hline Age $($ years $)$ & $25.8 \pm 3.75$ & $24.4 \pm 3.29$ & 0.471 \\
\hline Weight $(\mathrm{kg})$ & $70.4 \pm 6.67$ & $72.55 \pm 5.59$ & 0.255 \\
\hline Height $(\mathrm{cm})$ & $171.95 \pm 4.06$ & $172.6 \pm 3.69$ & 0.510 \\
\hline BMI $\left(\mathrm{kg} / \mathrm{m}^{2}\right)$ & $23.76 \pm 1.45$ & $21.01 \pm 1.50$ & 0.707 \\
\hline
\end{tabular}

$\mathrm{P}<0.05$

\section{Joint Position Sense (JPS)}

Evaluation of JPS of the ankle at $10^{\circ}$ and FROM $-5^{\circ}$ inversion target positions was done using active reproduction of active ROM. The outcome measure was mean absolute angular error (AAE). Results showed significant main effect of fatigue on JPS $(\mathrm{F}(3,36)=6.573, \mathrm{P}=0.001)$. Also, significant interaction between JPS and groups $(\mathrm{F}(3,36)=$ 3.932, $\mathrm{P}=0.016)$. Between groups comparisons showed non-significant difference at baseline. While, post fatigue evaluation showed significantly less AAE in KTG compared to CG at $10^{\circ}$ inversion. On the contrary, no significant difference between AAE for both groups at FROM $-5^{\circ}$. Within groups comparisons showed significantly higher AAE post fatigue compared to baseline in the control group at $10^{\circ}$, and FROM $-5^{\circ}$ inversion. Meanwhile, the difference at KT group for $10^{\circ}$ and FROM $-5^{\circ}$ inversion was not significant comparing baseline and post fatigue evaluations (table 2). The results indicated that the KT maintained the JPS at both targets after the fatigue protocol.

Table 2:- Comparison of mean (AAE) for volunteers in KTG and CG at pre and post fatigue evaluation at $10^{\circ}$ and FROM $-5^{\circ}$

\begin{tabular}{|c|c|c|c|c|}
\hline & \multicolumn{2}{|l|}{ Groups } & \multicolumn{2}{|c|}{ Pairwise comparisons } \\
\hline Evaluations & $\begin{array}{l}\text { KTG } \\
\text { Mean }(+ \text { SD })\end{array}$ & $\begin{array}{l}\text { CG } \\
\text { Mean }(+\mathrm{SD})\end{array}$ & F- Stat. & Sig. \\
\hline Baseline $10^{\circ}$ & $2.4(+1.19)$ & $2.21(+1.41)$ & 0.850 & 0.362 \\
\hline Post fatigue $10^{\circ}$ & $2.44(+1.26)$ & $3.56(+1.59)$ & 6.146 & $0.018 *$ \\
\hline $\begin{array}{r}\text { Pairwise comparisons } r \text { F-stat } \\
\text { Paired Sig. }\end{array}$ & $\begin{array}{l}0.142 \\
0.708\end{array}$ & $\begin{array}{l}11.626 \\
0.002 *\end{array}$ & & \\
\hline Baseline FROM $-5^{\circ}$ & $1.96(+1.33)$ & $2.17(+1.19)$ & 0.399 & 0.602 \\
\hline Post fatigue FROM $-5^{\circ}$ & $2.76(+1.54)$ & $3.84(+3.04)$ & 0.763 & 0.165 \\
\hline $\begin{array}{l}\text { Pairwise comparisons } \quad \text { F-stat } \\
\text { Paired Sig. }\end{array}$ & $\begin{array}{l}2.300 \\
0.138\end{array}$ & $\begin{array}{l}10.089 \\
0.003 *\end{array}$ & & \\
\hline
\end{tabular}

$* \mathrm{P}<0.05$ 


\section{Evertors peak torque (EPT)}

The statistical results showed significant main effect of fatigue on EPT $\left(\mathrm{F}_{(1,38)}=23.327, \mathrm{P}<0.001\right)$. While the interaction effect between EPT and groups was non- significant $\left(\mathrm{F}_{(1,38)}=0.377, \mathrm{P}<0.543\right)$. Pairwise comparisons showed significant reduction of EPT following fatigue in both KTG and CG. On the other hand, between groups pairwise comparisons showed non- significant difference between mean EPT both at baseline and post evaluations (Table 3).

\section{Discussion:-}

The aim of this study was to investigate the effects of KT on ankle proprioception, and isokinetic peak torque after evertor muscles fatigue. This would give a clue whether KT application to ankle evertors could help in preventing lateral ankle sprains.

Table 3:- Comparison of mean Eversion peak torque (EPT) for volunteers in KTG and CG at pre and post fatigue evaluation

\begin{tabular}{|l|l|l|l|l|}
\hline & Groups & \multicolumn{2}{l|}{ Pairwise comparisons } \\
\hline \multirow{2}{*}{ Evaluations } & KTG & CG & F- Stat. & Sig. \\
\hline Pre fatigue EPT (Nm) & $16.39(3.73)$ & $16.81(4.49)$ & 0.106 & 0.747 \\
\hline Post fatigue EPT (Nm) & $13.76(3.34)$ & $13.42(4.72)$ & 0.069 & 0.794 \\
\hline Pairwise comparisons F-stat & 8.885 & 14.819 & & \\
Paired Sig. & $0.005^{*}$ & $0.000^{*}$ & & \\
\hline
\end{tabular}

$* \mathrm{P}<0.05$

\section{Joint position sense (JPS)}

The results of the study showed that using KT maintained the ankle JPS following the fatigue protocol at $10^{\circ}$ and FROM $-5^{\circ}$ inversion positions in KTG. On the contrary, ankle JPS significantly decreased at both angles following fatigue in the control group. AAE compared post fatigue was significantly lower in the KTG at $10^{\circ}$ inversion compared to CG. These results suggest that the application of the KT might be beneficial to maintained the ankle JPS if applied before the football match or training.

Bjorklund et al. (2001) suggested that fatigue may influence the mechanoreceptors in the muscles around the ankle joint, thus adversely affects ankle proprioception. It is suggested that KT helps skin to stretch as much as possible by creating enough space between the skin and the muscles. Such stretching enhances proprioception through increasing the stimulation of cutaneous mechanoreceptors (Chang et al., 2010; Halseth et al., 2004; Kase and Kase, 2003 ).

The results of this study indicate that there was no significant difference in AAE between KT and control groups at FROM $-5^{\mathrm{O}}$ following fatigue protocol. Normally close to the end range of joint motion stretching of the skin and stimulation of cutaneous mechanoreceptors takes place (Riemann and Lephart, 2002). Thus, the postulated mechanism by which KT enhances proprioception would be available for players in both groups near the end range. Accordingly, this might has closed the gap between the reported AAE in both groups at FROM $-5^{0}$ inversion. This finding was supported by a recent study who investigated the effect of KT on ankle proprioception in 26 individuals who had experienced ankle sprain (Seo et al., 2016). The study assessed joint position sense during dorsiflexion/ plantarflexion and inversion/eversion. The study found that joint position sense after Kinesio taping was improved immediately in the dorsiflexion and inversion positions. On contradiction to the results of the present study, a previous RCT investigated the effect of KT on ankle proprioception in 68 healthy individuals immediately after KT application and 48 hours later and found that KT did not improve the ankle proprioception (Miralles et al., 2014). Differences from current study may result from different KT application, difference in time of measurements, and presence of fatigue protocol in the current study. Briem et al. (2011) analyzed the effect of KT compared to nonelastic sports tape in 51 male athletes. The author found non-elastic sports tape better in improving dynamic ankle muscles' support. Further, they suggested that efficacy of KT is unlikely to prevent ankle sprain via the same mechanism. However, this study was a repeated measure crossover study under different conditions. It was not clear in their report whether they allowed a wash out time or not. Further, effect of fatigue in the current study may be a source of difference. Furthermore, in another contradicting study, Halseth et al. (2004) concluded that KT did not change in proprioception at the ankle plantar flexion and plantar flexion with inversion in healthy subjects. 
Differences in outcomes compared to current study may be related to the application of fatigue protocol and participation of athletes in the current study. Besides, the difference in ankle positions during testing in both studies.

\section{Evertors Peak Torque (EPT)}

The results of this study suggested that EPT decreased significantly following fatigue in both KTG and CG, with no significant differences between mean EPT of both groups post fatigue. In the current study, it was hypothesized that applying KT with inhibition technique would minimize evertors activity during fatigue protocol. Thus, KT would likely reduce the effect of fatigue on evertors and maintain EPT. However, findings showed that KT was not able to maintain EPT following fatigue. The reason might be that the application of KT might had created a lower EPT baseline immediately following application, before fatigue protocol. Such possibility could not be verified in this study as it lacked an immediate assessment of EPT following application and before fatigue. Either way, EPT nonsignificant difference between KTG and CG post fatigue suggests that the application of KT would not achieve the required outcome. The current findings are supported by the result of the study by Strutzenberger et al. (2016) who investigated whether gluteal KT might enhance strength of lower limbs muscles with or without fatigue in rugby players. They demonstrated that following muscles fatigue KT was not able to influence the leg's explosive strength. Likewise, Yeung et al. (2015) conducted an investigation about the effect of KT on peak torque of the knee extensors following fatigue. They reported that the peak torque was not maintained following fatigue protocol. On the other hand, study's findings came inconsistent with the study of Ahn et al. (2015) who found that KT was effective in restoring the peak torque of quadriceps muscle after muscle fatigued induction of 45 healthy participants. Authors in Ahn's study applied KT extensively over vastus medialis, vastus lateralis, and rectus femoris. They attributed their results to facilitation of motor function due to cutaneous afferent stimulation. Therefore, extensive cutaneous stimulation might cause their effects in contrast to current study.

\section{Conclusion:-}

Results of this study suggest that KT could be helpful in preventing lateral ankle sprains in football players. This likely effect could be achieved through maintaining ankle JPS in inversion, rather than maintaining EPT, following evertor muscles fatigue.

\section{Conflict of Interest:-}

The authors declare that they have no conflict of interest.

\section{References:-}

1. Ahn, I. K., Kim, Y. L., Bae, Y. H. and Lee, S. M. (2015). Immediate Effects of Kinesiology Taping of Quadriceps on Motor Performance after Muscle Fatigued Induction. Evid Based Complement Alternat Med, 410526(10), 2.

2. Ament, W. and Verkerke, G. J. (2009). Exercise and fatigue. Sports Med, 39(5), 389-422.

3. Bjorklund, M., Hamberg, J. and Crenshaw, A. G. (2001). Sensory adaptation after a 2-week stretching regimen of the rectus femoris muscle. Arch Phys Med Rehabil, 82(9), 1245-1250.

4. Briem, K., Eythorsdottir, H., Magnusdottir, R. G., Palmarsson, R., Runarsdottir, T. and Sveinsson, T. (2011). Effects of kinesio tape compared with nonelastic sports tape and the untaped ankle during a sudden inversion perturbation in male athletes. J Orthop Sports Phys Ther, 41(5), 328-335.

5. Chang, H. Y., Chou, K. Y., Lin, J. J., Lin, C. F. and Wang, C. H. (2010). Immediate effect of forearm Kinesio taping on maximal grip strength and force sense in healthy collegiate athletes. Phys Ther Sport, 11(4), 122-127.

6. Dabadghav, R. (2016). Correlation of ankle eversion to inversion strength ratio and static balance in dominant and non-dominant limbs of basketball players. J Sports Med Phys Fitness, 56(4), 422-427.

7. Drouin, J. M., Valovich-mcLeod, T. C., Shultz, S. J., Gansneder, B. M. and Perrin, D. H. (2004). Reliability and validity of the Biodex system 3 pro isokinetic dynamometer velocity, torque and position measurements. Eur J Appl Physiol, 91(1), 22-29.

8. Fayson, S. D., Needle, A. R. and Kaminski, T. W. (2015). The Effect of Ankle Kinesio Tape on Ankle Muscle Activity During a Drop Landing. J Sport Rehabil, 24(4), 391-397.

9. Fong, D. T., Chan, Y. Y., Mok, K. M., Yung, P. S. and Chan, K. M. (2009). Understanding acute ankle ligamentous sprain injury in sports. Sports Med Arthrosc Rehabil Ther Technol, 1(14), 1758-2555.

10. Fong, D. T., Hong, Y., Chan, L. K., Yung, P. S. and Chan, K. M. (2007). A systematic review on ankle injury and ankle sprain in sports. Sports Med, 37(1), 73-94. 
11. Fu, T. C., Wong, A. M., Pei, Y. C., Wu, K. P., Chou, S. W. and Lin, Y. C. (2008). Effect of Kinesio taping on muscle strength in athletes-a pilot study. J Sci Med Sport, 11(2), 198-201.

12. Gabbett, T. J. (2000). Incidence, site, and nature of injuries in amateur rugby league over three consecutive seasons. Br J Sports Med, 34(2), 98-103.

13. Gonzalez-Iglesias, J., Fernandez-de-Las-Penas, C., Cleland, J. A., Huijbregts, P. and Del Rosario GutierrezVega, M. (2009). Short-term effects of cervical kinesio taping on pain and cervical range of motion in patients with acute whiplash injury: a randomized clinical trial. J Orthop Sports Phys Ther, 39(7), 515-521.

14. Gray, H. (2000). Anatomy of the Human Body (20th ed edition ed.). Bartleby.

15. Halseth, T., McChesney, J. W., Debeliso, M., Vaughn, R. and Lien, J. (2004). The effects of kinesio taping on proprioception at the ankle. J Sports Sci Med, 3(1), 1-7.

16. Hootman, J. M., Dick, R. and Agel, J. (2007). Epidemiology of collegiate injuries for 15 sports: summary and recommendations for injury prevention initiatives. J Athl Train, 42(2), 311-319.

17. Hsu, Y. H., Chen, W. Y., Lin, H. C., Wang, W. T. and Shih, Y. F. (2009). The effects of taping on scapular kinematics and muscle performance in baseball players with shoulder impingement syndrome. J Electromyogr Kinesiol, 19(6), 1092-1099.

18. Kase, K. (2005). Illustrated Kinesio Taping (Fourth edition ed.). Ken'I-Kai, Tokyo, Japan.

19. Kase, K., Wallis, J. and Kase, T. (2010). Clinical Therapeutic Applications of the Kinesio Taping Method (2 ed.). Kinesio, USA.

20. Kase, W. J. and Kase, T. (2003 ). Therapeutic Applications of the Kinesio Taping Method. Ken'I-kai, Tokyo, Japan.

21. Larsson, B., Kadi, F., Lindvall, B. and Gerdle, B. (2006). Surface electromyography and peak torque of repetitive maximum isokinetic plantar flexions in relation to aspects of muscle morphology. $\mathrm{J}$ Electromyogr Kinesiol, 16(3), 281-290.

22. Lephart, S. M., Pincivero, D. M. and Rozzi, S. L. (1998). Proprioception of the ankle and knee. Sports Med, 25(3), 149-155.

23. Martin, R. L., Davenport, T. E., Paulseth, S., Wukich, D. K. and Godges, J. J. (2013). Ankle stability and movement coordination impairments: ankle ligament sprains. J Orthop Sports Phys Ther, 43(9).

24. McLoda, T. A., Stanek, J. M., Hansen, A. J. and McCaw, S. T. (2009). A task failure has no effect on the electromechanical delay of the peroneus longus. Electromyogr Clin Neurophysiol, 49(2-3), 109-115.

25. McNair, P. J., Depledge, J., Brettkelly, M. and Stanley, S. N. (1996). Verbal encouragement: effects on maximum effort voluntary muscle action. Br J Sports Med, 30(3), 243-245.

26. Miralles, I., Monterde, S., del Rio, O., Valero, S., Montull, S. and Salvat, I. (2014). Has Kinesio tape effects on ankle proprioception? A randomized clinical trial. Clinical Kinesiology: Journal of the American Kinesiotherapy Association, 68(2), 9-18.

27. Mohammadi, F. and Roozdar, A. (2010). Effects of fatigue due to contraction of evertor muscles on the ankle joint position sense in male soccer players. Am J Sports Med, 38(4), 824-828.

28. Porter, T. and Rushton, A. (2015). The efficacy of exercise in preventing injury in adult male football: a systematic review of randomised controlled trials. Sports Med Open, 1(1), 014-0004.

29. Riemann, B. L. and Lephart, S. M. (2002). The Sensorimotor System, Part II: The Role of Proprioception in Motor Control and Functional Joint Stability. J Athl Train, 37(1), 80-84.

30. Seo, H.-D., Kim, M.-Y., Choi, J.-E., Lim, G.-H., Jung, S.-I., Park, S.-H., Cheon, S.-H. and Lee, H.-Y. (2016). Effects of Kinesio taping on joint position sense of the ankle. Journal of physical therapy science, 28(4), 11581160.

31. Strutzenberger, G., Moore, J., Griffiths, H., Schwameder, H. and Irwin, G. (2016). Effects of gluteal kinesiotaping on performance with respect to fatigue in rugby players. Eur J Sport Sci, 16(2), 165-171.

32. Subasi, V., Cakir, T., Arica, Z., Sarier, R. N., Filiz, M. B., Dogan, S. K. and Toraman, N. F. (2016). Comparison of efficacy of kinesiological taping and subacromial injection therapy in subacromial impingement syndrome. Clin Rheumatol, 35(3), 741-746.

33. Tankevicius, G., Lankaite, D. and Krisciunas, A. (2013). Test-retest reliability of biodex system 4 pro for isometric ankle-eversion and -inversion measurement. J Sport Rehabil, 22(3), 212-215.

34. Waterman, B. R., Owens, B. D., Davey, S., Zacchilli, M. A. and Belmont, P. J., Jr. (2010). The epidemiology of ankle sprains in the United States. J Bone Joint Surg Am, 92(13), 2279-2284.

35. Willems, T., Witvrouw, E., Verstuyft, J., Vaes, P. and De Clercq, D. (2002). Proprioception and Muscle Strength in Subjects With a History of Ankle Sprains and Chronic Instability. J Athl Train, 37(4), 487-493.

36. Wilson, E. L. and Madigan, M. L. (2007). Effects of fatigue and gender on peroneal reflexes elicited by sudden ankle inversion. J Electromyogr Kinesiol, 17(2), 160-166. 
37. Woods, C., Hawkins, R., Hulse, M. and Hodson, A. (2003). The Football Association Medical Research Programme: an audit of injuries in professional football: an analysis of ankle sprains. Br J Sports Med, 37(3), 233-238.

38. Yeung, S. S., Yeung, E. W., Sakunkaruna, Y., Mingsoongnern, S., Hung, W. Y., Fan, Y. L. and Iao, H. C. (2015). Acute effects of kinesio taping on knee extensor peak torque and electromyographic activity after exhaustive isometric knee extension in healthy young adults. Clin J Sport Med, 25(3), 284-290. 